RU-97-75

UTTG-24-97

PUPT-1719

IASSNS-HEP-97/101

hep-th/9709091

\title{
Schwarzschild Black Holes from Matrix Theory
}

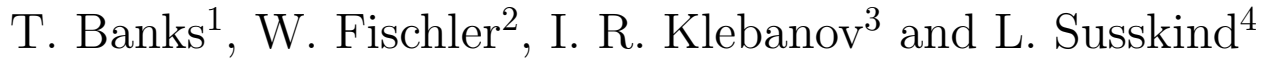

\begin{abstract}
We consider Matrix theory compactified on $T^{3}$ and show that it correctly describes the properties of Schwarzschild black holes in $7+1$ dimensions, including the energy-entropy relation, the Hawking temperature and the physical size, up to numerical factors of order unity. The most economical description involves setting the cut-off $N$ in the discretized light-cone quantization to be of order the black hole entropy. A crucial ingredient necessary for our work is the recently proposed equation of state for $3+1$ dimensional SYM theory with 16 supercharges. We give detailed arguments for the range of validity of this equation following the methods of Horowitz and Polchinski.
\end{abstract}

September 1997

\footnotetext{
${ }^{1}$ Serin Physics Labs, Rutgers University, Piscataway, NJ 08855.

${ }^{2}$ Physics Department, University of Texas, Austin, TX 78712.

${ }^{3}$ Joseph Henry Laboratories, Princeton University, Princeton, New Jersey 08544.

${ }^{4}$ Institute for Advanced Study, Princeton, New Jersey 08540. Permanent address: Physics Department, Stanford University, Stanford, CA 94305.
} 


\section{Introduction}

The problem of extreme and near-extreme black holes in string theory has recently received a great deal of attention [1, 2, 3]. The quantum theory of D-branes [4, 5] and its relation to Supersymmetric Yang-Mills (SYM) theory [6] has allowed for successful qualitative (and sometimes quantitative) calculations of the properties of these objects. By contrast, little has been written about the Schwarzschild black holes in string theory, although a rough understanding has been achieved in [7, 8].

In this paper we take up the problem of Schwarzschild black holes in Matrix theory [9]. We will see that in the particular case of $7+1$ non-compact dimensions, enough is known about the relevant SYM theory to derive the properties of black holes, including the energyentropy relation and the physical size, up to numerical factors of order unity. In what follows, such numerical factors will be ignored throughout the paper.

Matrix theory is best thought of as the Discretized Light-Cone Quantization (DLCQ) of M-theory [10], i.e. compactification on a light-like circle of radius $R$. Accordingly, the longitudinal momentum $P_{-}=P^{+}$is quantized in integer multiples of $1 / R$,

$$
P_{-}=\frac{N}{R}
$$

We may further compactify $d$ transverse coordinates on a $d$-dimensional torus. For simplicity, we will often consider this torus to be "square" with equal circumferences given by $L$. Another length scale that appears in the theory is the 11-dimensional Planck length, $l_{11}$.

The Matrix theory conjecture is that the sector of the theory with given value of $N$ is exactly described by $U(N)$ SYM theory in $d+1$ dimensions with 16 real supercharges. This theory lives on a dual torus with circumferences [11, 12]

$$
\Sigma \sim \frac{l_{11}^{3}}{R L}
$$

For physical applications the limit $N \rightarrow \infty$ has to be taken. This limit is not uniform in the following sense: if we ask how large $N$ must be taken in order to achieve a given degree of accuracy, the answer will depend on the system under investigation. However, choosing $N$ too large can introduce a needlessly large number of degrees of freedom, most of which may be frozen into their ground state. The situation is in many respects similar to the choice of cut-off in quantum field theory, where it is desirable to choose it so that there are neither too few nor too many degrees of freedom. The former destroys the accuracy, while the latter makes the calculations unnecessarily difficult.

The minimal value $N_{\min }$ which will allow the desired degree of accuracy for a black hole will certainly increase with the entropy which, after all, is the measure of the number of relevant degrees of freedom. Our first task will be to determine $N_{\min }$. Consider a black hole in its rest frame. The transverse momentum $P_{\perp}=0$, while $P_{+}=P_{-}=M$. The transverse size of the black hole is its Schwarzschild radius, $R_{s}$, and its extension in the $X^{-}$direction 
is also of order $R_{s}$. As $R_{s}$ grows, it will eventually exceed the light-like compactification scale $R$, and the black hole will not fit in the longitudinal space. However, we may boost it, thereby Lorentz contracting it, until it does fit. Let us assume that it is boosted till its longitudinal momentum is $N / R$. Its longitudinal size is then contracted to

$$
\Delta X^{-} \sim \frac{M}{P_{-}} R_{s}=\frac{M R}{N} R_{s}
$$

The condition for fitting into the transverse space is $R>\Delta X^{-}$, which implies

$$
N>M R_{s}=N_{\min }
$$

Thus we see that simple kinematical considerations determine the order of magnitude of $N_{\min }$.

The Schwarzschild radius in a $D$-dimensional space-time is

$$
R_{s} \sim\left(G_{D} M\right)^{\frac{1}{D-3}}
$$

where $G_{D}$ is the $D$-dimensional Newton constant. Thus, (4) becomes

$$
N_{\min } \sim G_{D}^{\frac{1}{D-3}} M^{\frac{D-2}{D-3}}
$$

It is extremely interesting that the above expression is also the Bekenstein entropy of the black hole, $N_{\min } \sim S$.

Our strategy for determining the entropy-mass relation for the black hole goes as follows. Using the Matrix theory hamiltonian $H$ for fixed $N$ we compute the partition function, $Z=\operatorname{Tr} e^{-\beta H}$. From this we deduce the relation between the energy and the entropy for given $N$,

$$
E=E(N, S)
$$

Next we observe that the Matrix theory hamiltonian is identified with the DLCQ energy according to

$$
E=\frac{M^{2}}{P_{-}}=\frac{M^{2} R}{N}
$$

Thus, we find

$$
M^{2}=\frac{N}{R} E(N, S)
$$

Now, for $N \gg N_{\text {min }}$, the value of $M^{2}$ computed this way must be independent of $N$. However, as we shall see, computing the partition function for $N \gg S$ is very difficult. Thus, we are forced to choose $N \sim S$, and (9) becomes

$$
M^{2} \sim \frac{S}{R} E(S, S)
$$

Note that the Matrix hamiltonian is explicitly proportional to $R$, so that $R$ cancels in (10) leaving a relation between mass and entropy. 


\section{The case $d=3$}

One might have guessed that the easiest case to analyze is that of $D=11$ black holes, where the matrix model is just supersymmetric quantum mechanics. In fact, this case seems to be especially difficult: very little is known about this system for general $N$. The case involving the most widely studied SYM theory is $d=3$, leading to $D=8$ black holes. Therefore, we concentrate on the $d=3$ case.

The SYM theory relevant to the $D=8$ black holes is the very special self-dual conformally invariant theory in $3+1$ dimensions with 16 real supercharges. We will begin by illustrating the strategy outlined in the previous section without fully justifying the formulae. More details are given in the next section.

Since the SYM theory is conformally invariant, its equation of state must have the form

$$
S=C \Sigma^{3} T^{3}, \quad E=C \Sigma^{3} T^{4},
$$

where $T$ is the temperature of the Matrix theory (not to be confused with the Hawking temperature), and $\Sigma^{3}$ is the volume of the dual torus. $C$ measures the number of degrees of freedom which, for the adjoint representation of $U(N)$, is expected to be $C \sim N^{2}$. This equation of state is supported by the form of the near-extremal entropy of the self-dual 3 -brane found in 13, 14.

Eliminating the temperature from (11) and using (2), (8) gives

$$
S \sim M^{3 / 2}\left(\frac{G_{11}}{N L^{3}}\right)^{1 / 4} .
$$

Now we set $N \sim S$ and use the standard expression for the Newton constant in 8 dimensions, $G_{8}=G_{11} / L^{3}$, arriving at

$$
S \sim M^{6 / 5} G_{8}^{1 / 5}
$$

which is correct for $D=8$ black holes! Note that not only does the scaling with $M$ come out correctly, but so does the dependence on $L / l_{11}$.

Although the above derivation will prove to be correct, there are serious questions concerning the range of validity of (11). At the point $S \sim N$, the temperature given by (11) with $C \sim N^{2}$ satisfies

$$
\Sigma T \sim 1 / N^{1 / 3}
$$

For a conventional free field with periodic boundary conditions the equation of state (11) is valid only when the temperature satisfies $\Sigma T>1$. This is just the condition that the wavelength of a typical thermal quantum is smaller than the box size. Clearly, (14) requires us to extrapolate the equation of state to much lower temperatures. This sort of situation has arisen before in the theory of D-brane black holes [15, 16] where, due to the presence of Wilson loops, the effective size of the quantization box is much larger than its actual size. We will return to this point in the next section and show that this is exactly what happens when a single 3 -brane is wrapped $N^{1 / 3}$ times over each of the direction of the 3 -torus. 
Before doing this, however, let us consider implications of black hole physics for the equation of state when $N \gg S$ or, equivalently, $\Sigma T \ll 1 / N^{1 / 3}$. In this range the entropy must be independent of $N$ for given $M$. In $D=8$ the formula is $S=M^{6 / 5} G_{8}^{1 / 5}$. Using $M^{2}=E N / R$ and $d E=T d S$ we find the equation of state

$$
S=(N T \Sigma)^{3 / 2} .
$$

At the point $S=N$ this agrees with our previous equation of state. The implication is that the eqation of state (11) which holds at high temperature must continue down to temperature

$\sim \frac{1}{N^{1 / 3} \Sigma}$ but no further. A transition to the equation of state (15) must occur at this point. We will see in the next section that there is good reason to believe that just such a transition occurs.

\section{Thermodynamics of wrapped 3-branes}

In this section we study the thermodynamics of Dirichlet 3-branes wrapped over a rectangular 3-torus with sides of length $\Sigma_{i}$. We will be particularly interested in a single 3-brane wrapped $N_{1}$ times over direction $\hat{1}, N_{2}$ times over direction $\hat{2}$, and $N_{3}$ times over direction $\hat{3}$. The coordinates along such a 3 -brane are given by

$$
X^{i}=\frac{N_{i} \Sigma_{i}}{2 \pi} \theta_{i}, \quad i=1,2,3
$$

where $\theta_{i}$ are the three angles running from 0 to $2 \pi$. The total volume of such a multiply wound 3 -brane is

$$
V_{\text {tot }}=N_{1} N_{2} N_{3} \Sigma_{1} \Sigma_{2} \Sigma_{3} .
$$

Therefore, its charge is the same as that of $N=N_{1} N_{2} N_{3}$ singly wound parallel 3-branes. The dynamics of such a system is governed by $\mathcal{N}=4$ supersymmetric $U(N)$ Yang-Mills theory in $3+1$ dimensions [6]. To describe the multiply wound configuration, appropriate Wilson loops need to be introduced [17]. For example, a D-string wound $N_{1}$ times is described in $1+1$ dimensional SYM theory by the holonomy which is a shift matrix: its non-zero entries are $\phi_{i+1, i}=1$ for $i=1, \ldots, N_{1}-1$ and $\phi_{1, N_{1}}=1$. In other words, the holonomy matrix encodes how the different strands of the D-string are connected. Similarly, the three $U(N)$ holonomy matrices for the multiply wound 3-brane encode the connections among the $N_{1} N_{2} N_{3}=N$ sheets as we move along the holonomy cycles.

For sufficiently large $\Sigma_{i}$ (or the temperature $T$ ) there should be no difference between the thermodynamic properties of the multiply wound brane and those of $N$ coincident singly wound branes. The latter theory has $O\left(N^{2}\right)$ massless degrees of freedom on volume $V_{d}=$ $\Sigma_{1} \Sigma_{2} \Sigma_{3}$, and we find the following expressions for the energy and the entropy,

$$
E \sim N^{2} V_{d} T^{4}, \quad S \sim N^{2} V_{d} T^{3} .
$$


For the multiply wound brane the same scalings follow from a different line of reasoning, which is based on the arguments in [8]. Now the fields live on the volume $V_{\text {tot }}=N V_{d}$, and there are $O(N)$ massless species. The latter fact may seem surprising, but it is a direct consequence of the D-brane theory [4, 5, 17]. Indeed, the 3-brane consists of $N=N_{1} N_{2} N_{3}$ interconnected sheets, and there are distinct massless open strings connecting sheet 1 with sheet $j, j=1, \ldots, N$.

The difference between the two configurations is illuminated by T-dualizing along all three directions. The $N$ singly wound 3-branes are mapped into $N$ coincident 0 -branes on the dual torus. The multiply wound 3-brane is instead mapped into an array of 0-branes [18], with $N_{1}$ rows along direction $\hat{1}, N_{2}$ rows along direction $\hat{2}$, and $N_{3}$ rows along direction $\hat{3}$. A string connecting two 0-branes in general has a fractional winding number along direction $i$, quantized in units of $1 / N_{i}$. For each allowed winding number, we find $N$ different species because the string can start on each of the 0-branes in the array. This implies that, before T-duality, the allowed values of momentum in the $i$-th direction are quantized in units of $2 \pi /\left(N_{i} \Sigma_{i}\right)$, and we have $N$ different massless fields.

While for high enough temperature it does not matter how the $N 3$-branes are interconnected, a crucial difference appears as the temperature is lowered. For $N$ singly wound branes, (18) holds approximately only if $T \Sigma_{i}>1$. For the multiply wound brane, the momenta $p_{i}$ are quantized in units of $2 \pi /\left(N_{i} \Sigma_{i}\right)$, and the condition on the temperature is much less restrictive,

$$
T N_{i} \Sigma_{i}>1
$$

Let us assume that all three $N_{i} \Sigma_{i}$ are comparable. Then we find that the lowest temperature at which (18) applies is

$$
T_{\text {crit }} \sim\left(V_{d} N\right)^{-1 / 3}
$$

At this temperature, the entropy $S$ is of order $N$. Thus, we can achieve adequate resolution of the black hole $\left(N \sim N_{\text {min }}\right)$ right at the edge of the range of validity of (18). This fact is of crucial importance for describing the properties of 8-dimensional Schwarzschild black holes in the context of Matrix theory.

$T_{\text {crit }}$ is the temperature of the black hole in the boosted frame. Let us calculate the value of the Hawking temperature by boosting $T_{\text {crit }}$ back to the rest frame of the black hole. We find

$$
T_{H} \sim \frac{N}{R M}\left(V_{d} N\right)^{-1 / 3} \sim \frac{S^{2 / 3}}{M G_{8}^{1 / 3}}
$$

Using

$$
M \sim S^{5 / 6} G_{8}^{-1 / 6}
$$

we have

$$
T_{H} \sim\left(S G_{8}\right)^{-1 / 6} \sim \frac{1}{R_{s}}
$$

This is indeed the expected scaling of the Hawking temperature. 
Another connection of the $3+1$ dimensional $\mathcal{N}=4$ supersymmetric $U(N)$ Yang-Mills theory is with the semiclassical properties of the R-R charged 3-branes in type IIB supergravity. This connection has been explored in considerable detail in [13, 14, 19, 20, 21]. For example, the ADM energy and the Bekenstein-Hawking entropy are given in terms of the Hawking temperature by relations of the form (18) [13, 14]. For infinite 3-branes, these relations hold down to $T=0$, but in the finite case we know that there is a minimal temperature below which they break down. What is the origin of such a restriction from the point of view of the classical solution? The geometry which corresponds to the multiply wrapped brane is

$$
d s^{2}=f^{-1 / 2}\left(-h d t^{2}+d y^{i} d y^{i}\right)+f^{1 / 2}\left(h^{-1} d r^{2}+r^{2} d \Omega_{5}^{2}\right),
$$

where

$$
f(r)=1+\frac{r_{3}^{4}}{r^{4}}, \quad h(r)=1-\frac{r_{0}^{4}}{r^{4}} .
$$

We will consider the near-extremal case where $r_{0} \ll r_{3}$, and $r_{3}$ is related to $N$ through [13, 19]

$$
r_{3}^{4} \sim N g\left(\alpha^{\prime}\right)^{2} .
$$

Absence of large corrections to the metric from the higher-derivative terms in the string effective action requires that $N g>1$ [19]. Furthermore, for the case of finite 3-branes we will require that the longitudinal volume at the horizon is at least of order 1 in string units, i.e.

$$
V_{d} \frac{r_{0}^{3}}{r_{3}^{3}}>\left(\alpha^{\prime}\right)^{3 / 2} .
$$

In terms of the temperature $T \sim r_{0} / r_{3}^{2}$, this condition becomes

$$
V_{d} T^{3}>(N g)^{-3 / 4} \text {. }
$$

Even for large $N g$ this condition is more restrictive than $V_{d} T^{3}>1 / N$ found for the multiply wound case. Thus, there is no contradiction between the SYM theory and the classical calculations. The SYM approach indicates that (18) does not apply for $T<T_{\text {crit }} \sim\left(N V_{d}\right)^{-1 / 3}$, but the classical solution receives potentially large $\alpha^{\prime}$ corrections starting at a higher temperature. Assuming agreement with SYM, we conjecture that in reality the $\alpha^{\prime}$ corrections stay small all the way down to $T \sim T_{\text {crit }}$.

\section{Estimating the size of the black hole}

Let us estimate the size of a $D=8$ black hole. As we explained, this is well described by a uniform array of $N$ 0-branes on the original 3-torus of volume $V$. The rms size of the

black hole should be identified with $\sqrt{\left\langle\vec{X}^{2}\right\rangle}$ where $\vec{X}$ is the transverse position of one of the 0-branes. 
By the virial theorem, the kinetic energy of the 0-branes scales as the total energy in the system,

$$
\frac{M_{0}}{2}\left\langle\sum_{i=1}^{N}\left(\frac{d}{d t} \vec{X}_{i}\right)^{2}\right\rangle \sim N^{2} V_{d} T^{4} .
$$

Since $M_{0}=1 / R$, we find that for each 0 -brane

$$
\left\langle\left(\frac{d}{d t} \vec{X}\right)^{2}\right\rangle \sim R N V_{d} T^{4} .
$$

Using the fact that the typical frequency is of order $T$, we conclude that

$$
\left\langle\vec{X}^{2}\right\rangle \sim R N V_{d} T^{2} .
$$

Now we recall that $T_{\text {crit }} \sim\left(N V_{d}\right)^{-1 / 3}$, a nd that $V_{d}$, the volume of the dual torus, is $G_{11} /\left(R^{3} V\right)$. Substituting into (31) we find that $R$ cancels out, as it should, and

$$
\left\langle\vec{X}^{2}\right\rangle \sim\left(N G_{11} / V\right)^{1 / 3} .
$$

Since the Newton constant in $D=8$ is $G_{8}=G_{11} / V$, we finally have甲

$$
\sqrt{\left\langle\vec{X}^{2}\right\rangle} \sim\left(S G_{8}\right)^{1 / 6} .
$$

This is precisely the scaling with the entropy of the Schwarzschild radius in $D=8$ ! It is remarkable that this scaling follows so simply from the Matrix theory.

We have seen that the 0 -branes spread out over the 3-torus undergo large transverse oscillations. As a result of these oscillations, some number of them can break off the metastable bound state and be emitted into the transverse directions. This is the physical picture of the Hawking radiation. Let us estimate the typical number of 0-branes in the emitted cluster. In the rest frame of the black hole, the typical values of $p_{0}$ and $p_{z}$ for massless Hawking particles are expected to be of order $1 / R_{s}$. Thus, $p_{-}^{\text {rest }} \sim 1 / R_{s}$ also. Now we boost this value to the Matrix theory frame, where the total $P_{-}$of the black hole is $N / R$. In this frame the typical value for a Hawking particle is

$$
p_{-} \sim \frac{N}{R M} \frac{1}{R_{s}} \sim \frac{1}{R} .
$$

This means that Hawking emission proceeds a few 0-branes at a time. Eventually the black hole completely dissociates into small clusters of 0-branes.

\section{Acknowledgments}

We are grateful to Steve Shenker for useful discussions. The work of I.R.K was supported in part by the DOE grant DE-FG02-91ER40671, the NSF Presidential Young Investigator

\footnotetext{
${ }^{5}$ The same scaling follows if we work on the dual torus and estimate the thermal fluctuations of scalar fields in the SYM theory.
} 
Award PHY-9157482, and the James S. McDonnell Foundation grant No. 91-48. L.S. is grateful to E. Witten for hospitality at the IAS where part of this work was done. L.S. is a Raymond and Beverly Sackler Fellow at IAS and is supported in part by NSF grants PHY-9219345 and PHY-9513835. T.B. is supported in part by the DOE grant DE-FG0296ER40559. W.F. is grateful to Rutgers University for hospitality while part of this work

was being done. W.F. is supported in part by the NSF under grant PHY-95-11632 and by the Robert A. Welch Foundation.

\section{References}

[1] A. Sen, Nucl. Phys. B440 (1995) 421.

[2] A. Strominger and C. Vafa, Phys. Lett. B379 (1996) 99.

[3] For a review, see J. Maldacena, Princeton University Ph.D. Thesis, hep-th/9607235.

[4] J. Dai, R. G. Leigh, and J. Polchinski, Mod. Phys. Lett. A4, 2073 (1989);

R. G. Leigh, Mod. Phys. Lett. A4, 2767 (1989).

[5] J. Polchinski, Phys. Rev. Lett. 75, 4724 (1995).

[6] E. Witten, Nucl. Phys. B460 (1996) 541.

[7] L. Susskind, hep-th/9309145.

[8] G. Horowitz and J. Polchinski, Phys. Rev. D55 (1997) 6189, hep-th/9612146.

[9] T. Banks, W. Fischler, S. Shenker and L. Susskind, Phys. Rev. D55 (1997) 5112, hep-th/9610043.

[10] L. Susskind, hep-th/9704080.

[11] L. Susskind, hep-th/9611164.

[12] O. Ganor, S. Ramgoolam and W. Taylor, Nucl. Phys. B492 (1997) 191.

[13] S.S. Gubser, I.R. Klebanov and A.W. Peet, Phys. Rev. D54 (1996) 3915, hepth/9602135.

[14] I.R. Klebanov and A.A. Tseytlin, Nucl. Phys. B475 (1996) 165, hep-th/9604089.

[15] J.M. Maldacena and L. Susskind, Nucl. Phys. B475 (1996) 679, hep-th/9604042.

[16] S. Das and S. Mathur, Phys. Lett. B375 (1996) 103.

[17] J. Polchinski, S. Chaudhuri and C. Johnson, hep-th/9602052. 
[18] A. Hashimoto, hep-th/9610250.

[19] I.R. Klebanov, Nucl. Phys. 496 (1997) 231, hep-th/9702076.

[20] S.S. Gubser, I.R. Klebanov and A.A. Tseytlin, Nucl. Phys. B499 (1997) 217, hepth/9703040.

[21] S.S. Gubser and I.R. Klebanov, hep-th/9708005. 